\title{
Aprendizaje basado en problemas en el camino a la innovación en ingeniería
}

\author{
José Eucario Parra Castrillón \\ Ingeniero de Sistemas \\ Magister en Desarrollo de Software \\ Magister en Educación \\ Eucarioparra5@gmail.com
}

\author{
Mauricio José Amariles Camacho \\ Ingeniero Electrónico \\ Master Network and Information \\ security \\ mauricio.amariles@usbmed.edu.co
}

\author{
Carlos Arturo Castro Castro \\ Ingeniero de Petróleos \\ Especialista en Sistemas de \\ Información Geográfica \\ carlos.castro@usbmed.edu.co
}

(Tipo de Artículo: Reflexión. Recibido el 22/06/2016. Aprobado el 12/07/2016)

\begin{abstract}
Resumen. Una buena opción para promover la creatividad y la innovación es implementar procesos formativos que partan del análisis de problemas contextualizados. En esta vía, en vez de un enfoque centrado en los contenidos de un curso, la propuesta es diseñar ambientes de aprendizaje que motiven a los estudiantes a recrearse con situaciones complejas de la cotidianeidad. Bajo esta concepción de escenarios de Aprendizaje Basados en Problemas (ABP), en la Facultad de Ingeniería de la Universidad de San Buenaventura - Sede Medellín -, desde 2013 se viene implementando una iniciativa que ha motivado el desarrollo de distintas estrategias docentes. Es así como bajo este marco se han implementado distintas estrategias para que los estudiantes creativamente propongan soluciones a las situaciones problemáticas. Lo anterior merece resaltarse si se tiene en cuenta que esas estrategias están inmersas dentro de un currículo tradicional centrado en asignaturas y unos procesos de evaluación clásicos que determinan técnicas e indicadores puntuales a veces sin contexto. En los párrafos siguientes se exponen las condiciones y formas como se ha desarrollado el ABP y asimismo, cuál ha sido su contribución a los propósitos pedagógicos para la innovación.
\end{abstract}

Palabras clave. Aprendizaje Basado en Problemas, ABP, Creatividad, Competencias Propositivas, Competencias Argumentativas, Competencias Interpretativas, Enseñanza de la Ingeniería, Innovación, Tipos de Problemas.

\section{Problem based learning on the road to innovation in engineering}

\begin{abstract}
A good choice for promoting creativity and innovation is to implement educational processes based on the analysis of application problems. In this way, instead of focusing on the content of a course approach, the proposal is to design learning environments that encourage students to recreate with complex situations of everyday life. Under this scenario conception problembased learning (PBL) in the Faculty of Engineering of the University of San Buenaventura - Medellin - since 2013 is being implemented an initiative that has motivated the development of different teaching strategies. Thus, under this framework they have been implemented various strategies for students propose creative solutions to problematic situations. This should be emphasized if one takes into account that such strategies are embedded within a traditional curriculum focused on subjects and processes that determine classic evaluation techniques and specific indicators sometimes without context. In the following paragraphs the conditions and methods are disclosed as ABP has been developed and also, what was their contribution to the educational purposes for innovation.
\end{abstract}

Keywords. Argumentative Skills, Based Learning, Creativity, Engineering Education, innovation, Interpretative Skills, PBL, Purposeful Skills, Types of Problems.

\section{Introducción}

Una de las preocupaciones en las facultades de ingeniería está relacionada con la forma como se deben llevar las metodologías de enseñanza, para que realmente en los estudiantes se logren aprendizajes significativos y competencias. Se entiende que se deben formar ingenieros para su desempeño en la aplicación de la tecnología, la técnica, y la ciencia, en la creación de innovaciones y la solución de problemas. Dentro de tal perspectiva resulta esencial el conocimiento matemático y el razonamiento lógico para la formulación de modelos coherentes con los requisitos, necesidades, intereses y el contexto de las intervenciones.

En relación con tales propósitos de la ingeniería, se conciben históricamente modelos de enseñanza que se enmarcan dentro de la rigurosidad y la catedra formal, donde sobresalen las formas rígidas de evaluación. $\mathrm{Si}$ bien esas metodologías son exitosas, al evidenciarse como se progresan exponencialmente en materia de infraestructura y calidad de procesos y servicios empresariales, dos inquietudes vienen sobrecogiéndose en la comunidad académica: las características del estudiante actual y las exigencias demandas por las vertiginosas cotidianeidades.
En el primer aspecto, el estudiante actual, como parte de un contexto global, intercomunicado y con tendencia a la movilidad, se manifiesta polifacético y con capacidades para la ubicuidad (puede hacer varias cosas al mismo tiempo, sin que se limiten sus capacidades). Con respecto a la cotidianeidad, pareciera que el mundo impulsa a la gente a hacer rápidamente lo mejor y lo más práctico. Tanto lo uno como lo otro, en sus consistencias internas, pueden reñir con las metodologías clásicas de la ingeniería.

De esta forma pareciera que la enseñanza de la ingeniería está en el medio de dos tensiones. Desde un lado la cientificidad y el rigor metodológico como requisitos intrínsecos; desde el otro, la flexibilidad y la intercomunicación con el contexto real de los estudiantes. No obstante, los reclamos de la sociedad por las competencias del ingeniero para la innovación en productos, infraestructuras y procesos que creen beneficio social y económico, no pierden vigencia. Asimismo, las exigencias de ingenieros competentes para la creatividad sigue siendo un asunto prioritario.

Como alternativa metodológica de enseñanza se propone el Aprendizaje Basado en Problemas - ABP porque es coherente con el rigor per se de la ingeniería, es adecuado con la naturaleza flexible del estudiante 
actual y es un impulsor pedagógico para la creatividad (requisito para la innovación). La metodología ABP se puede aplicar de distintas formas, aun dentro de diseños curriculares clásicos. Así, se evidencia en el Programa de Ingeniería de Sistemas de la Facultad de Ingeniería de la Universidad de San Buenaventura Medellín, donde se evoluciona al respecto, desde la cristalización de proyectos de investigación, la capacitación docente y la puesta en acción en los ambientes de aprendizaje.

\section{Conceptos acerca del ABP}

Dentro del marco del Aprendizaje Basado en Problemas - $\mathrm{ABP}$-, lo fundamental está en los ambientes de aprendizaje creados desde situaciones problematizadoras pertinentes y significativas para los estudiantes [1]. "Durante la solución del problema los estudiantes están aprendiendo los contenidos programados y luego desde la solución final, los estudiantes vuelven sobre lo aprendido, tanto acerca del ámbito del problema mismo, como del método de solución"[2].

En [3] se define el ABP como una orientación de la instrucción y el currículo centrado en la acción del estudiante, que lo lleva a investigar, aplicar teoría y poner en acción habilidades para conseguir una solución viable de un problema concreto. En su esencia el $A B P$ determina diseño curricular y orientación pedagógica centrada en la actividad del estudiante, quien utiliza situaciones y problemas de la vida real para alcanzar aprendizajes significativos. Por su parte, en [4] se afirma que es una estrategia que se apoya en el planteamiento de un problema para estimular en los estudiantes el desarrollo y adquisición de conocimiento. A su vez, [5] afirma que es un método de instrucción para que los estudiantes aprenden resolviendo problemas enmarcados dentro de un problema más complejo que puede tener varias respuestas.

Las anteriores concepciones se conectan en tres aspectos: el problema como punto de partida de la instrucción; el estudiante entendido como el actor central los aprendizajes; la pedagogía activa motivadora de la indagación del estudiante. Como resultado los conocimientos y competencias se logran en la medida que los estudiantes hacen investigaciones para encontrar soluciones a los problemas planteados.

Según lo anterior, el problema propiamente dicho determina la ruta a seguir en el proceso formativo. Por eso surgen entonces inquietudes acerca de los problemas. Esto es, ¿Qué encierra un problema con intencionalidad pedagógica? ¿Los problemas son los que están ya planteados o hay que diseñarlos? ¿Los problemas son reales o son hipotéticos? ¿Cuáles son las condiciones acerca de la complejidad del problema?

Al respecto [6] y [7] plantean alternativas: a) Llevar problemas sencillos a la clase para mostrar conceptos específicos b) Llevar a la clase un problema más complejo que el anterior, estructurado y con la información necesaria para resolverlo c) Proporcionar un problema complejo, no estructurado, que requiere de información adicional que deben conseguir los propios estudiantes. d) Proporcional el mismo problema anterior, pero planeando una fase adicional de análisis de la solución y de reflexión de información o conocimiento adicional que su puede utilizar para resolver el problema. Se deduce que esta última opción tiene un mayor aporte para el aprendizaje significativo y la adquisición de competencias interpretativas, propositivas y argumentativas.

Según [8] y [9], pueden presentarse problemas estructurados y no estructurados. Los primeros requieren aplicar un determinado conjunto de conceptos, técnicas, reglas, algoritmos, formulas 0 principios para resolver una situación problematizada. Además, claramente se conoce información de entrada, el resultado esperado, la delimitación y metodológicamente el profesor entrega la información al estudiante. A su vez, los problemas no estructurados se caracterizan porque en el planteamiento se desconocen algunos elementos, la información de entrada puede ser incompleta, pueden tener una o varias soluciones, probablemente conllevan a resolverse de distintas maneras con criterios diversos, obligan al planteamiento de hipótesis distintas o alternativas de solución, presentan delimitación difusa y motivan a la expresión de juicios de los estudiantes.

También con respecto a la relación entre ABP y los problemas, [10] plantean que los problemas deben ser no estructurados y motivar la libre indagación de los estudiantes y además, se deben promover actitudes de colaboración como parte de la metodología. Al respecto, es de anotarse que el trabajo en equipo con criterio colaborativo es pieza fundamental del ABP. Asimismo, se destaca que la metodología es interactiva, centrada en el estudiante, organizado para trabajar en grupos pequeños, y metódicamente orientada por el profesor, quien adquiere el modo de facilitador en el ambiente de aprendizaje. De esta forma, en el ABP el inicio de la clase no es expositivo, por el contrario, esta se dinamiza desde la movilización de los estudiantes para identificar los aprendizajes que se necesitan para resolver un problema [11].

El ABP es abierto y contextualizado, según [2]. "La metodología $A B P$ hace parte de un concepto de aprendizaje general: los entornos de aprendizaje abiertos (EAA). Algunas características de estos son los siguientes: asientan procesos con problemas para la interpretación y la experimentación; enlazan problemas complejos con experiencias cotidianas; sitúan planteamientos heurísticos, flexibles y de perspectivas múltiples; conectan la cognición con el contexto de modo inextricable: establecen modelos de entendimiento desde el realice de los errores" [12] citado por [2].

Además, se plantea en el mismo artículo que "Para el logro de los objetivos del aprendizaje, los EAA plantean tres métodos: a) Definido externamente, introduciendo al estudiante en la solución de un problema concreto, por ejemplo, crear un sistema para automatizar la conducción de un tren. b) Generado externamente, 
introduciendo al estudiante en un problema general sin particularizar el objetivo o la tarea, por ejemplo, sistemas para optimizar rutas de distribución. c) Creado de forma individual, introduciendo al estudiante en un dominio concreto (un caso), por ejemplo, conocer el origen de cierto virus informático" [2].

Como alternativa del ABP está el aprendizaje basado en proyectos. Puede decirse que esta es una modalidad de $A B P$, pero hay que enfatizar en una diferencia fundamental: el ABP basado en una situación problematizadora tiene su propósito fundamental en el aprendizaje, método para resolver el problema y en el aprendizaje de los conceptos explorados. En cambio, cuando la base es el desarrollo de un proyecto el énfasis está en las condiciones del producto final y la efectividad de la intervención. No obstante, en ambos casos son requisitos comunes la conexión con la cotidianeidad de los estudiantes y la motivación alta de estos para emprender procesos creativos. "Una forma de desarrollar ABP, es el aprendizaje basado en proyectos. Puede considerarse que esta es una modalidad de pedagogía activa que permite comprender el ámbito real del desempeño profesional. El estudiante emprende un acercamiento a la realidad a partir de proyectos sobre problemas reales. El aprendizaje a partir de proyectos se caracteriza por la formación del razonamiento aplicado a contextos reales; al búsqueda de contenidos en función de su uso en la práctica; la puesta en escena del aprendizaje en dominios de naturaleza cotidiana "[2].

\section{Pedagogía para la innovación en la ingeniería}

Las apuestas por una ingeniería innovadora es la tendencia de la misma carrera tecnológica. Así lo plantea [13], quien afirma que la sociedad no se conforme con el diseño de máquinas, sino que además la creatividad debe apuntarle a las maquinas inteligentes, entendiéndose que ser creativo es pieza fundamental para comprender y desarrollar habilidades y capacidades para interpretar experiencias precedentes y proponer unas nuevas. Bajo estos conceptos del citado autor, se concibe la creatividad es la capacidad del ser humano para desarrollar innovación transformadora, vivacidad imaginativa, visión mental organizada, flexibilidad de pensamiento y originalidad imaginaria.

Como puede deducirse, ser creativo es requisito para ser innovador y de acuerdo los conceptos precedentes, la innovación es una facultad para el buen ejercicio de la ingeniería.

Además, la creatividad tal como la platea Torres, tiene unos atributos como espontaneidad, flexibilidad y asociación cognitiva y como proceso evidencia fases como la generación de ideas, su depuración posterior, la interpretación y agrupación de ideas, el planteamiento y evaluación de soluciones.

La creatividad, el ingenio, la innovación, la solución efectiva de problemas, son propósitos adjuntos al ideal de la ingeniería. El reto para las facultades es como formar esos ingenieros y cuáles son los ambientes educativos que se requieren crear. Especialmente el tema de la innovación viene convirtiéndose en un asunto sustancial para las empresas y por eso para las universidades.

En Castro y Fernández [14] mencionan cinco formas diferentes de innovación: a) La introducción de un nuevo producto; b) La introducción de un nuevo proceso; c) La apertura de un mercado nuevo; d) La adopción de una nueva materia prima; e) La implementación de una nueva forma de organización empresarial. En todas ellas un factor común es la creatividad e iniciativa para proponer novedades. Pero además, es requisito que la innovación en cualquiera de estas sea útil para las empresas o para la sociedad, es decir, que tenga sentido práctico y resuelva problemas reales.

Con respecto a las personas innovadoras, [15] afirman que "quienes participan en la generación de ideas $e$ innovaciones deben tener creatividad $e$ imaginación, ser capaces de conectar ideas, tener curiosidad, dotes de observación, capacidad para experimentar, pero también necesitan interés para abordar y resolver problemas".

La ingeniería tiene una ideología y valores que conducen su accionar, siendo el gusto por la innovación su expresión más relumbrante. "La ingeniería hay que entenderla como un proyecto cognitivo siempre en curso, inacabado". Estas ideas las expresa [16] en torno al espíritu creador de los ingenieros. Acá se plantea una analogía entre los artistas y los ingenieros innovadores, en el sentido que sus misiones están encauzadas hacia la belleza pero no el ornamento. De esta forma se distingue la cualidad original artística, de la neta aplicación de conocimientos técnicos para revolver problemas específicos.

Con respecto a la innovación, [17] la sustenta como el arte y capacidad para creación de valor económico a partir de los cambios en lo estético, lo paradigmático o lo tecnológico. La intuición mental del ingeniero es fundamental dentro de la industria de la innovación, al igual que su creatividad y su inteligencia. Tal intuición, entendida como sabiduría informal llega hasta a superar dogmas cuando los ingenieros desafían incluso a principios paradigmáticos de arte o ciencia. Es decir, los procesos mentales de los ingenieros generan productos satisfactorios que cumplen con las especificaciones, sin que necesariamente sus procederes puedan reducirse a razonamientos secuenciales y lógicos. Intuición, arte e ingeniería están estrechamente ligadas. "la innovación representa la ideología de la discontinuidad creativa dentro de la ingeniería" [18].

En consideración de tal marco conceptual de la ingeniería, cabe preguntarse si las personas nacen con perfiles de ingeniería (creativos e innovadores) o si por el contrario, son el resultado de ambientes educativos y sociales. En relación con esto, se afirma que la creatividad dialécticamente oscila entre las dimensiones internas y externas de las personas [19] Lo interno es la manifestación genética de potencialidades, habilidades, 
aptitudes y capacidades (creatividad heredada). Lo externo hace referencia a las interacciones en el plano socio-cultural, con grupos formales e informales. Las oportunidades sociales le permiten al ser humano incrementar las potencialidades heredadas, según lo concluyen estos autores.

Varios autores, proponen laboratorios virtuales para mejorar la enseñanza en ingeniería y optimizar recursos como es el caso del laboratorio para automatización industrial [20] y el laboratorio de instrumentación electrónica [21].

Según lo anterior es apropiado considerar una pedagogía para la innovación en ingeniería. Esto es, planear estrategias para ambientes educativos que fomenten la creatividad de los estudiantes. La opción que se propone es el $\mathrm{ABP}$, en consideración del estudiante activo e investigador que se va formando en la medida que asume retos para resolver problemas.

Una hipótesis es que si hacen diseños metodológicos acordes con las concepciones de ABP, se da la oportunidad a los estudiantes de imaginar soluciones, estudiar casos, plantear alternativas y descartar otras, trabajar en equipos solidariamente, construir rutas de indagación y acercarse al espíritu emprendedor. También como hipótesis podría afirmarse que el ABP determina en los estudiantes de ingeniería actitudes, aptitudes y competencias para la creatividad y la innovación.

\section{Implementación del ABP en Ingeniería de Sistemas}

En el Programa de Ingeniería de Sistemas de la Universidad de San Buenaventura - Medellín - , se implementó $A B P$ con tres estrategias: a) ABP desde la planeación de varios proyectos dentro de un mismo grupo de estudiantes (propuesta de colectivos de proyectos); b) ABP desde la planeación de un proyecto general de ingeniería aplicada; c) ABP desde el surgimiento de proyectos enlazados a las pre-conceptos de los estudiantes. En las tres formas se han tenido como propósitos el fortalecer las competencias propositivas, interpretativas y argumentativas de los estudiantes.

No obstante, en consideración de los avances de la metodología, se pasó a las siguientes alternativas: a) Planteamiento, planeación, desarrollo y evaluación de un único problema durante todo el semestre b) Planteamiento, planeación, desarrollo y evaluación de varios problemas durante todo el semestre c) Desarrollo de un proyecto de investigación aplicada durante todo el semestre d) Exploración, estudio y análisis de uno o varios casos del tipo empresarial o científico. En todas las alternativas anteriores, el problema puede tener la forma de estudio de caso, proyecto, enunciado hipotético o enunciado real. Además, el docente en esas opciones asume el rol de planeador, facilitador y evaluador de los procesos y de los resultados.
Se ha considerado en el Programa de Ingeniería de Sistemas, que en la concepción de ABP se da la posibilidad a la realización de proyectos. El planteamiento sobre el aprendizaje basado en proyectos como una alternativa de $A B P$, se justifica desde el problema mismo, el cual, dentro de la lógica para la planeación, desarrollo y evaluación de proyectos, es la base fundamental. Un proyecto adquiere forma cuando hay el reconocimiento de un problema para ser resuelto metódicamente en una organización de actividades secuenciales.

Varias son las apreciaciones que han justificado esta iniciativa. En primer lugar, el análisis ha partido de la inquietud acerca de cómo deben ser los procesos de enseñanza, en momentos en los que se habla de una crisis de la ingeniería, en cuanto a la demanda de estudiantes, ya que pareciera que las nuevas generaciones tienen otras expectativas. Esta preocupación además se hace más tensa con realidades como la dificultad cognitiva que implican las ciencias básicas, la tardanza para que los estudiantes de los primeros semestres entren en interacciones con los objetos propios de la Ingeniería de Sistemas o la insuficiencia de los laboratorios con respecto a las tecnologías empresariales.

Segundo, se sabe que en el momento histórico del siglo XXI se evidencian transformaciones en las formas como los estudiantes asumen sus actividades académicas. En este sentido, las metodologías de enseñanza, deberían adecuarse para estar en coherencia con la mentalidad de los nuevos sujetos, con sus marcos de practicidad, elasticidad, paralelismo, movilidad y la ubicuidad. Pero estos criterios pueden reñir con la concepción clásica de la ingeniería, ya que por ejemplo para [22], la ingeniería es el campo o la disciplina, práctica, profesión y arte que se relaciona con el desarrollo, adquisición y aplicación de la técnica, la ciencia y el conocimiento matemático en la comprensión, diseño, desarrollo, invención, innovación y el uso de materiales, máquinas, estructuras, sistemas y procesos para fines específicos. Se debe buscar entonces una pedagogía vinculada con estas dos tensiones y que reconozca concepciones en uno y otro lado.

Tercero, los estudiantes de Ingeniería de Sistemas navegan en la realidad de unos contextos ineludibles. Por ejemplo, el paradigma de la flexibilidad sociocultural, la incorporación tecnológica en los modos de actuación de las personas, las inclinaciones por el facilismo, las preferencias por el agilísimo o el deterioro del concepto de erudición por preferir el de la practicidad. Esto determina llevar al aula ambientes educativos apropiados para ese contexto general y que, además, impulsen la determinación del estudiante para su creatividad.

Cuarto, es común el reclamo del sector empresarial por unos ingenieros con capacidades técnicas y con sentido práctico para resolver problemas aplicando la teoría adecuadamente. Tal practicidad se asocia con la mentalidad creativa y con las actitudes para innovar. 
Ingenierías USBMed, Volumen 7, No. 2, Julio-Diciembre 2016

Estas competencias pueden alcanzarse si la planeación curricular se piensa desde la dinamización del aula, a partir de actividades que fomenten la acción creativa de los estudiantes.

En cuanto a la fundamentación y aspectos curriculares, en primera instancia para implementar ABP se ha invitado a los profesores al reconocimiento de la viabilidad del problema planteado, su conveniencia con respecto al nivel cognitivo del grupo de estudiantes, e incluso, con respecto a las emociones y expectativas, aparte de otras circunstancias como el tiempo y los recursos y de detalles en cuanto a la forma, la sintaxis, la semántica y el léxico. Es de anotar que estas dinámicas se hacen internamente dentro de los grupos, aun con la estructura curricular basada en asignaturas y la destinación fija de tiempos, espacios y créditos académicos.

En relación con la evaluación, en la metodología $A B P$ los propósitos están acordes con la dinámica del estudiante desde una mirada integral. Por esto se deben proponer acciones pedagógicas e indicadores de evaluación continua sobre competencias para el saber conocer, saber ser y saber hacer [22] y que se pueden superponer con la tipología de competencias interpretativas, propositivas y argumentativas. Las competencias en la categoría del saber ser hacen referencia al comportamiento en el contexto; competencias las de la categoría del saber conocer tienen que ver con la conceptualización de acuerdo con un plan de estudios; las competencias en el saber hacer están dirigidas a la solución de problemas con los recursos y conceptos disponibles.

Las competencias propositivas se articulan con las capacidades del estudiante para plantear hipótesis, explicar determinados problemas, o hacer búsquedas de alternativas frente a un problema (Es aquella acción que persigue que el estudiante proponga alternativas que puedan aplicarse en un contexto determinado). "Las competencias argumentativas se reflejan en la capacidad de la persona para exponer los problemas y las soluciones; las propositivas están en relación con sus actitudes para plantear hipótesis y soluciones; las competencias interpretativas se relacionan con la comprensión del sentido de los conceptos y su naturaleza" [23], citado por [2].

\section{Implementación del ABP}

En el Proyecto Educativo del Programa de Ingeniería de Sistemas de la Universidad de San Buenaventura, se plantea que el desarrollo de las actividades formativas se orienta en el paradigma metodológico de Aprendizaje Basado en Problemas - ABP - , sin exclusión de otras formas de aprendizaje implicadas por las conceptualizaciones teóricas y los formalismos lógicos. Como enfoques para el aprendizaje se consideran también el aprendizaje de casos y el desarrollo de proyectos.

Un avance importante es la intervención del micro currículos del programa de Ingeniería de Sistemas. Se logró en cada asignatura la declaración de argumentativas, propositivas e interpretativas que el estudiante debe alcanzar y la construcción de una guía ABP donde se especifican el tipo de situación problematizadora que se va a desarrollar, los recursos, la bibliografía y los indicadores para las competencias. Es de anotar que en este caso se evitaron formatos excesivamente detallados y especificaciones exhaustivas de criterios de desempeño 0 de funcionalidades.

\subsection{ABP desde las propuestas de colectivo de proyectos}

En ocho grupos de Introducción a la Ingeniería (entre 2014 y 2015) se ha aplicado ABP con la participación de 120 estudiantes. Al interior de cada grupo se forman equipos de trabajo y en cada uno se propone un proyecto distinto. Como instrumento de motivación el proceso se acompaña con un documento sobre innovación tecnológica. Los roles del profesor consisten en: a) Presentación de la guía de elaboración del proyecto b) Organización de exposiciones de los estudiantes c) Preparación de lluvias de ideas y d) Asesoría a cada uno de los proyectos presentados.

"Varios aspectos se destacan en estos dos grupos. El primero y el más importante es la conexión de los estudiantes con la especificidad de las ingenierías Electrónica, Multimedia, Industrial, de Sonido y de Sistemas, pues los grupos de Introducción a la Ingeniería son interdisciplinarios. Obviamente, la profundidad de los proyectos llega hasta la delimitación conceptual de estudiantes de primer semestre y el producto que entregan se reduce es a un anteproyecto, pero aun así, ellos presentan unas ideas que sirven de canal para conectarse con las temáticas propias de su ingeniería. Es en este caso cuando se plantean la formación de sus competencias argumentativas. Además, los estudiantes deben exponer sus avances, indagaciones, dificultades y propósitos y por esto se fortalecen las competencias propositivas $e$ interpretativas. Un segundo aspecto destacado es la alta motivación que presentan los estudiantes y el afán que demuestran para hacer lo mejor. Su esfuerzo es manifiesto en las exposiciones, en el trabajo en equipos que organizan, en la adaptación de los conceptos y en general, en la alegría que sienten por la conexión con la especificidad de sus carreras." [2].

Con respecto a la evaluación, se tuvieron en cuenta el avance de las propuestas de los estudiantes en cuanto a sus capacidades para la argumentación en el momento exponer las características de la innovación que proponen, la interpretación del problema que resuelven y la proposición de alternativas de solución, para elegir la más viable. Todo esto, dentro de las limitaciones metodológicas y conceptuales que pueden observarse en estudiantes del primer semestre de universidad. 


\subsection{ABP desde la planeación de un proyecto general de ingeniería aplicada}

Una experiencia importante de ABP fue la integración curricular flexible que se alcanzó en la siguiente situación:

"La metodología ABP se aplicó en el año 2014 desde un proyecto general para todos los estudiantes de cuatro asignaturas integradas en un solo grupo: Programación Orientada a Objetos, Programación Orientada a Servicios, Informática II y Programación Avanzada, de las carreras Ingeniería de Sistemas, Ingeniería Multimedia e Ingeniería Electrónica.

El proceso fue dirigido por un docente principal y un docente acompañante. Ambos tomaron el rol de facilitadores en la formación de sus estudiantes. Desde el primer día de clase, el docente planteó una situación real y común, para el desarrollo de un proyecto de software. El punto de partida fue la presentación a los estudiantes de unos requisitos funcionales y no funcionales para el desarrollo de un aplicativo Web que preste servicios de automatización en la gestión de las facturas de servicios domiciliarios. Los requerimientos se consignan en un documento que se entrega de forma individual a cada estudiante, con el fin de motivar la discusión y elección en consenso de un diseño para la implementación del software [2].

En una fase posterior cada equipo de estudiantes programa por separado su propios sistema, aunque todos atienden al mismos análisis y diseño. Es decir, en el grupo al final resultaron varios sistemas de información, uno por cada equipo, pero resolviendo el mismo problema, con iguales especificaciones de requisitos funcionales y no funcionales y atendiendo a un mismo diseño arquitectónico.

En 2015 de nuevo se implementó esta estrategia de ABP, pero el problema se cambió. Esta vez el análisis, diseño y desarrollo fue para un sistema de información para la gestión de proyectos de grado.

"Los temas transversales que se han desarrollado tanto en 2014 como en 2015, han sido técnicas de programación orientada a objetos, técnicas de programación orientada a servicios, frameworks y lenguajes de programación. Además, los estudiantes avanzan conceptualmente conocimientos sobre especificación de requisitos, aplicación de diagramas de clases y de diagramas de casos de uso " [2], temas que no son propiamente de los cursos de fundamentos y lenguajes de programación y que sirven de abrebocas para las asignaturas de ingeniería del software.

Esta misma estrategia de ABP se aplicó en Ingeniería del Software I (ingeniería de requisitos), Ingeniería del Software II (diseño y arquitectura del software) e Ingeniería de Software III (calidad, verificación y validación de software). Estas tres asignaturas están conectadas en su metodología y aplicación, de manera que la situación problematizadora avanza entre los distintos grupos y en cada uno de ellos los equipos de trabajo proponen las soluciones dentro del ciclo de vida del software en las fases correspondientes. Un asunto para destacar es que los docentes de estas asignaturas están en plena cooperación y hablando el mismo idioma.

\subsection{ABP desde el surgimiento de proyectos enlazados a las pre-conceptos de los estudiantes}

En esta modalidad no hay inicialmente un proyecto planeado. En consenso con los estudiantes y de acuerdo con sus experiencias previas y pre-conceptos, se decide sobre el tipo de realización. Esta modalidad se aplicó en 2014 en la asignatura Seminario de Nuevas Tecnologías (Sistema de Información Geográfica). En 2015 se ha proseguido con este enfoque, pero se ha ampliado a las asignaturas de Seguridad Informática y Proyectos Informáticos (electiva profesional de Ingeniería de Sistemas).

Como orientación general en el caso de Sistemas de Información Geográfica, el profesor al iniciar el semestre académico le presenta al grupo de estudiantes distintos casos reales de ciudad que requieren de la aplicación de una plataforma informática de georreferención. A partir de esos problemas se promueve el aprendizaje de temas como cartografía, geodesia, bases de datos espaciales y geoinformática. En este caso los temas transversales se relacionan con los sistemas de información geográfica: funciones, bases de datos, programación de soluciones y problemas de localización, condiciones, tendencias, rutas, pautas y modelos.

En el caso de la materia Fundamentos de Programación (primer semestre ingenierías Multimedia, Industrial, Electrónica, Ambiental, de Sistemas y de Sonido), se ha adaptado el ABP en cada grupo, dependiendo de los pre-conceptos diagnosticados. Es así como en algunos grupos se ha propuesto el desarrollo de juegos aplicando la herramienta de programación Pseint (http://pseint.sourceforge.net/), mientras que en otros se ha hecho énfasis en la solución de problemas sobre áreas, volúmenes, movimiento y modelamiento matemático de situaciones cotidianas.

Un avance importante en las tres formas de ABP descritas tienen que ver con la incorporación de la Plataforma Virtual de Ingeniería, que ha servido como recurso de apoyo docente para que estudiantes y profesores puedan hospedar archivos, presentar avances y compartir recursos.

\section{Apoyo con ambientes virtuales}

Las experiencias de aplicación de ABP en el programa de Ingeniería de Sistemas, han sido apoyadas por un ambiente virtual (Plataforma Virtual de Ingeniería), que tiene como objetivos hospedar información y servir de canal de divulgación para los estudiantes. La plataforma tiene aulas virtuales para distintas asignaturas de los programas de Ingeniería de Sistemas e Ingeniería Electrónica. Se ha encontrado 
que desde los ambientes virtuales y la aplicación educativa de las tecnologías de la información y la comunicación - TIC - se favorece el ABP: Como un logro importante, en el semestre 2015-1 había 24 asignaturas con apoyo virtual.

Desde la experiencia docente en la Fundación Universitaria Católica del Norte y el SENA y desde los análisis resultado de los proyectos de investigación "a) Estructuración, Diseño y Evaluación de Contenidos Interactivos a Partir de Prácticas Ágiles “ (2014) , b) "Ingeniería de Software para Desarrollar Objetos de Aprendizaje - ISDOA - (2012) y c) "Metodología de Desarrollo de Software para Objetos Virtuales de Aprendizaje - MESOVA - " (2011), realizadas por el Grupo de Investigación en Modelamiento y Simulación Computacional (GIMSC) de la Universidad de San Buenaventura Seccional Medellín, se ha concluido que el estudiante en la modalidad virtual aprende siguiendo una metodología que parte desde la solución de los problemas, proyectos y actividades que se le proponen. Es decir, parte de asas estrategias y no de los contenidos. Va estos en la medida que las soluciones se lo reclaman y se apoya en exploraciones en bibliográficas y en Internet, cuando la necesidad se lo demanda.

En la educación virtual hay ciertos aspectos generales que con el transcurso de su desarrollo se han venido cristalizando y que tienen que ver con la actitud y el perfil de los estudiantes y cuyo punto de partida es el aprendizaje sobre el análisis de contextos significativos, para plantearse y revolver problemas. Las actividades concretas al respecto, son la mejor guía para las rutas metodológicas en el ambiente virtual. Pero además de lo anterior, se percibe también la necesidad de perfiles docentes con inteligencia suficiente para diseñar las situaciones problematizadoras y para correlacionarlas con las tecnologías disponibles. Estos dos aspectos con el agregado de una organización de los dispositivos tecnológicos, curriculares, instruccionales y comunicacionales, son los que determinan el éxito de las propuestas educativas virtuales. Inclusive, en aspectos concernientes a la evaluación, hay consensos sobre la formulación y solución de problemas como la forma más pedagógica y segura para motivar el desempeño acertado de los estudiantes.

Para comprender la dimensión de las relaciones entre las tecnologías de la Información y Comunicación con el $A B P$, se debe analizar que estas herramientas informáticas para procesar , almacenar, resumir , recuperar, presentar y codificar la información, demarcan ciertos compromisos para hacerlas verdaderamente constructivas en los ambientes educativos. Tal vez el más importante es la convicción sobre su aplicación para roles constructivistas y comunicativos que buscan de manera sistémica la apropiación de nuevos saberes para aplicarlos en el análisis, solución y planteamiento de problemas, esto es, la construcción de marcos propositivos y comprensivos, que determinen no solo la solución particular de casos concretos, sino y lo más importante, el cuestionamiento sobre otros problemas derivados. De no ser así, la aplicación de las TIC en la educación, no pasaría de ser más que unos simples canales para transmitir información desde unas fuentes precisas y no descodificada por los receptores pasivos.

Las ventajas expuestas sobre la aplicación de las TIC y los ambientes virtuales para el desarrollo del $A B P$, se pueden llevar a la educación presencial como apoyo educativo. Así se viene haciendo en la propuesta presentada en este artículo, resaltándose además que aunque la Facultad de Ingeniería de la Universidad de San Buenaventura ha tenido fuertes criterios de formación con metodologías clásicas y rigurosas, en Ingeniería de Sistemas se ha logrado flexibilizar e innovar pedagógicamente con la aplicación de ABP y plataforma virtual para apoyar los procesos formativos presenciales.

\section{Conclusión}

Varios aspectos son fundamentales y comunes en las tres modalidades expuestas de ABP: a) El punto de partida de las asignaturas son situaciones problematizadoras y no secuencialidad temática. No obstante los contenidos y las competencias argumentativas, propositivas y propositivas planeados en el micro currículo se cubren finalmente. b) Los procesos de evaluación se desarrollan con base en las actividades y productos entregables a lo largo del semestre académico. No tiene importancia las pruebas puntuales. Es fundamental la evidencia del trabajo colaborativo, las exposiciones y las indagaciones de los estudiantes. c) Se ha evidenciado que las clases se hacen más descansadas y divertidas. El acercamiento a la cotidianeidad de los problemas eleva los niveles de motivación y de interés de los estudiantes. d) La actitud de los estudiantes hacia la creatividad es muy positiva, sus expectativas se abren y con decisión proponen innovaciones.

Las metodologías $A B P$ que se han venido implementando, muestran que es posible su aplicación aun con una estructura curricular tradicionalista, organizada por asignaturas, inflexible en la gestión de tiempos e inspirada en la concentración de temáticas.

Adicionalmente, el ABP puede convertirse en una estrategia para disminuir deserción estudiantil, ya que se observa una alta motivación y participación de los estudiantes en el desarrollo de las actividades.

A lo anterior se agrega que con la implementación de ambientes virtuales se abren mayores posibilidades para compartir información y se pueden crear escenarios para que estudiantes y docentes compartan sus experiencias. En el caso concreto del ABP el apoyo virtual se convierte en una oportunidad para potenciar el $A B P$, pues la concepción pedagógica de esta metodología está vinculada con las formas de aprendizaje en las plataformas de e-learning. 


\section{Trabajos futuros}

Un reto hacia el futuro es aplicar ABP pero en unos ámbitos educativos que se coordinen con otros que provengan de las empresas y las comunidades. Esto es, lograr que los estudiantes aprendan y adquieran competencias en la medida que el currículo de sus programas académicos esté enfilados hacia el estudio de intervención de problemáticas externas a las instituciones de educación superior.

Acorde con esto, en la Facultad de Ingeniería se vienen estableciendo convenios y realizando actividades que apuntan a una conexión real de empresas estatales y privadas, con los procesos curriculares, especialmente de Ingeniería de Sistemas.

Además de lo anterior, para fortalecer el ABP como metodología, un asunto importante es el apoyo con ambientes virtuales, la aplicación de contenidos digitales para el aprendizaje y la utilización de redes sociales.

En el Grupo de Investigación en Modelamiento y Simulación Computacional (GIMSC) se vienen adelantando proyectos al respecto del segundo reto: Ingeniería de Software para Desarrollar Objetos de Aprendizaje - ISDOA - [24] y Metodología de Desarrollo de Software para Objetos Virtuales de Aprendizaje MESOVA - [25]. Y un marco de trabajo que integra MESOVA e ISDOA denominado MARCODA[26].

\section{Referencias}

[1] CH. Reigeluth; J. Moore. La enseñanza cognitiva y el ámbito cognitiva. En: Diseño de la instrucción. Teorías y modelos. Madrid: Aula XXI Santillana, 2000.

[2] E. Parra; C. Castro; M. Amariles. "Casos de éxito de la aplicación de la metodología de aprendizaje basado en problemas ABP". IngEam No 1, pp. 12-23, 2014.

[3] J. R. Savery. (2006, marzo 14). Overview of Problem-based Learning: Definitions and Distinctions. Interdisciplinary Journal of Problem-Based Learning. [En línea] Disponible en: http://docs.lib.purdue.edu/cgi/viewcontent.cgi?article=1002\&cont ext=ijpbl.

[4] M. Savin-Baden; K. Wilkie. Problem-based Learning. Berkshire, England: Open University Press, 2006.

[5] C.E. Hmelo-Silver. "Problem-Based Learning: What and How Do Students Learn?". Educational Psychology Review, Vol. 16, No. 3, pp.235-266, 2004.

[6] H.S. Barrows; A.C. Kelson. Problem-based learning in secondary education and the problem-based learning institute. Springfield, Illinois: Problem-Based Learning Institute, 1995.

[7] J. Biggs. "Enhancing teaching through constructive alignment". Higher education, Vol 3, No 32, pp. 347-364, 1996.

[8] D.H. Jonassen. "Instructional design model for well-structured and ill-structured problem-solving learning outcomes". Educational
Technology: Research and Development, Vol. 1, No 45, pp. 6595, 1997.

[9] D.H. Jonassen. "Toward a design theory of problem solving". Educational Technology: Research and Development, Vol. 4, No 48, pp. 63-85, 2000.

[10] E. De Graat; A. Kolmos. "Characteristics of Problem-Based Learning". International Journal of Engineering Education, Vol 5, No 19, pp. 657-662, 2003.

[11] A. Escribano; A. Del Valle. El Aprendizaje Basado en Problemas: Una propuesta metodológica en Educación. Madrid: Narcea Ediciones., 2008.

[12] M. Hannafin; S. Land; K. Oliver. Entornos de aprendizaje abiertos: fundamentos, métodos y modelos. En: Diseño de la instrucción. Teorías y modelos. Madrid: Aula XXI Santillana, 2000.

[13] L. Torres. "Creatividad en los sistemas". Revista Ingeniería e Investigación, No. 52, 2003.

[14] E. Castro; I. Fernández. El Significado de Innovar. Madrid: Catarata, 2013.

[15] E. Castro; I. Fernández. El Significado de Innovar. Madrid: Catarata, 2013, p.91.

[16] C. Lanza. "La innovación es ingeniería pura. La creatividad de los ingenieros españoles entre el siglo de las luces y la era de Internet”. Revista de Obras Públicas, No 3449, p.2, 2004.

[17] C. Lanza. "La innovación es ingeniería pura. La creatividad de los ingenieros españoles entre el siglo de las luces y la era de Internet”. Revista de Obras Públicas, No 3449, 2004.

[18] C. Lanza. "La innovación es ingeniería pura. La creatividad de los ingenieros españoles entre el siglo de las luces y la era de Internet”. Revista de Obras Públicas, No 3449, p. 19, 2004.

[19] J. Zailes; A. Ridao; J. Robinson. Lanza. “¿Cómo educar la creatividad en el contexto actual?". Intangible Capital No 2, Vol. 0, No 2, 2004.

[20] Ariza L., Carlos. Y Amaya H., Darío. LABORATORIO REMOTO PARA LA ENSEÑANZA DE LA PROGRAMACIÓN DE UN ROBOT INDUSTRIAL. Revista Ingenierias USBMed, Vol. 2, No. 1, Ene-Jun 2011 Disponible http://web.usbmed.edu.co/usbmed/fing/v2n1/v2n1a7.pdf

[21] Mendoza, Bayron A., Meneses, Gustavo A., Correa, Mónica J. y Ocampo, Yudy A. LABORATORIO VIRTUAL PARA LA ENSEÑANZA DE INSTRUMENTACIÓN ELECTRÓNICA. Revista Ingenieria USBMed, Vol. 1, No. 1, Jul-Dic 2010 Disponible en:

http://web.usbmed.edu.co/usbmed/fing/v1n1/v1n1a9.pdf

[22] S. Tobón. Formación Basada en Competencias. Bogotá: Ecoe Ediciones, 2004.

[23] ICFES. Exámenes de calidad de la educación superior en ingeniería de sistemas. Bogotá: Grupo de Procesos Editoriales, 2008.

[24] Castro, C., Serna, M., \& Taborda, R. (2011). Ingeniería de Software para Desarrollar Objetos de Aprendizaje-ISDOA-. EATIS, 12, 347-353.

[25] E. Parra. "Propuesta de metodología de desarrollo de software para objetos virtuales de aprendizaje -MESOVA-.". Revista Virtual Universidad Católica del Norte, No 34, pp. 113-137, 2011. 1998.

[26] Castro, C. A., Parra, E., Perdomo, W., \& Lagunes, A. Una propuesta de Marco para la Construcción de Contenidos Digitales de Aprendizaje con prácticas ágiles MARCODA 